\title{
Performance Evaluation of Censoring-Enabled Systems for Sequential Detection in Large Wireless Sensor Networks
}

\author{
Mohammed Karmoose*, Karim G. Seddik ${ }^{\dagger}$ and Ahmed K. Sultan* \\ ${ }^{*}$ Department of Electrical Engineering, Alexandria University, Alexandria 21544, Egypt. \\ ${ }^{\dagger}$ Electronics Engineering Department, American University in Cairo, AUC Avenue, New Cairo 11835, Egypt. \\ email: m_h_karmoose@alexu.edu.eg, kseddik@aucegypt.edu, salatino@alumni.stanford.edu
}

\begin{abstract}
In this paper, we consider a sequential binary hypothesis testing framework in wireless sensor networks. We study the effect of sensor censoring on network performance in terms of the average error probability and average number of observations required until a global decision is made. The detection process is mathematically modeled as a random walk process with two absorbing barriers. We resort to Chernoff bound in order to find upper bounds on the error probabilities and the average stopping time. The main contribution of this paper is to prove that in a sequential binary hypothesis network where sensors send their hard decisions to the fusion center, censoring can enhance the network performance in comparison to non-censoring networks in certain SNR regimes. Numerical evaluation is provided to illustrate the gains achieved through censoring.
\end{abstract}

\section{INTRODUCTION}

Wireless sensor networks (WSNs) are vastly deployed nowadays in various applications, many of which require the monitoring and the detection of a certain phenomenon. Based on local observations made by the sensors, each sensor reports to a centralized Fusion Center $(F C)$ which is responsible for making a global decision regarding the presence or absence of this phenomenon. This readily maps to the problem of distributed detection [1]. Recently, much research has been directed toward distributed detection in WSNs.

In this paper, we revisit the concept of sensor "censoring" in distributed detection from a different yet rather interesting point of view. In specific, we consider sensor censoring as a means to enhance system performance, in addition to the energy savings that are well characterized in literature. Rago et. al. [2] first envisioned censoring as a means to save transmission energy by imposing transmission constraints on the sensor nodes. Results proved asymptotically [2] and later generally [3] that the optimal censoring region - in terms of minimum error probability - is confined to a single interval of the Log-Likelihood Ratio (LLR) of the local decisions, with the lower threshold equal to zero. System degradation in terms of error probability was demonstrated for different censoring regions, which shows the energy-performance tradeoff inherent to censoring systems. Intensive research has been therefore directed toward employing censoring in distributed detection WSNs, and we mention for brevity [4]-[8].

However, one key observation about most publications considering censoring in distributed detection is the assumption of local soft-decisions: sensors send their locally computed LLR values to the FC. Conversely, a hard-decision framework would mandate sensors to apply one-bit quantization to the locally computed LLRs prior to transmission, and therefore only local decisions are sent to the FC for global decision making. Hard-decision-based detection framework was considered in [8], [9] to characterize the achieved energy savings via employing censoring. However, it was proven in [10], [11] that censoring can also be employed in harddecision frameworks to enhance system performance in terms of error probability of global decision. Sensors are assumed to adopt a parallel topology, with a Time-Division-MultipleAccess TDMA scheme [10] or a Type-Based-Multiple-Access TBMA scheme [11] when communicating with the FC. In this context, our main contrariness with [10], [11] is considering a sequential distributed detection framework.

Sequential distributed detection was considered in previous works in the literature, for example in [1], [12], [13]. A FC polls the sensors in a sequential manner for their local observations, and the process continues until a global decision can be made by the FC. This framework differs substantially from the conventional hypothesis testing framework [6] in that sensors are polled for their local decisions only if the $\mathrm{FC}$ requests so in order to achieve an acceptable level of reliability in the decision. Inherently, sequential detection systems are proven to be superior to conventional detection systems in terms of energy consumption. By allowing sensor nodes to censor transmissions in a certain uninformative region, it is natural to witness an increment in the average delay incurred until a global decision is made. In this work, we employ censoring in sequential distributed detection framework as a means to enhance the performance of such systems. Performance in this context is characterized by the error probability in the global decision and the average incurred delay.

Our main contributions can therefore be summarized as follows: 1) We consider a sequential detection framework based on hard decisions, in which local decisions made by the sensors are polled by the FC until a global decision is made. We show that censoring can be used to enhance system performance in terms of error probability and average delay. 2) We provide analytical upper bounds on the average error probability in global decisions and the average number of observations required to obtain one.

The paper is organized as follows: in Section II we describe the system and data models used in our analysis. Section III provides the mathematical analysis of the underlying random process of both the conventional and censoring-enabled sys- 


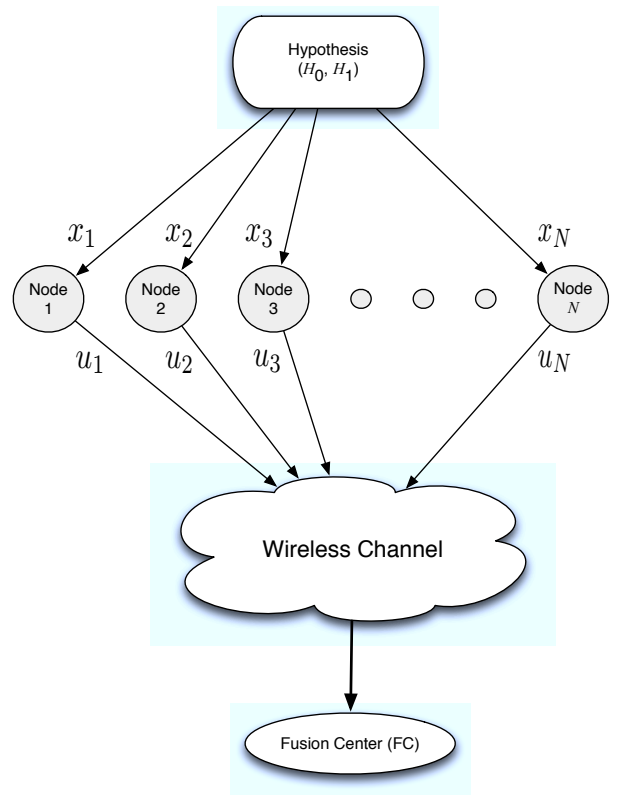

Fig. 1. System model

tems. Based on the obtained results, we provide upper bounds on the performance of these systems in Section IV. In Section $\mathrm{V}$ we highlight the main results deduced from the upcoming analysis via numerical evaluation, and we conclude the paper in Section VI.

\section{System Model}

We assume the presence of a sensor network that consists of an infinitely large number of identical sensors. The purpose of the network is to detect the presence or absence of a certain phenomenon. This maps to the classical binary hypothesis testing problem of detecting $H_{1}$ or $H_{0}$ respectively. A visual depiction of such a network is shown in Fig. 1. Each sensor makes a local decision regarding the current hypothesis based on its local observation $x_{\mathrm{n}}, \mathrm{n}=0,1,2, \ldots$ which is assumed to follow the following data model ${ }^{1}$

$$
\begin{aligned}
& H_{0}: x_{\mathrm{n}} \sim \mathcal{N}\left(0, \sigma_{0}^{2}\right) \\
& H_{1}: x_{\mathrm{n}} \sim \mathcal{N}\left(0, \sigma_{1}^{2}\right) .
\end{aligned}
$$

\section{A. Local Decisions}

Each sensor employs a log likelihood ratio test to make a local decision. The LLR of the jth sensor is given by

$$
l_{\mathrm{j}}\left(x_{\mathrm{j}}\right)=\log \left(\frac{\operatorname{Pr}_{1}\left(x_{\mathrm{j}}\right)}{\operatorname{Pr}_{0}\left(x_{\mathrm{j}}\right)}\right),
$$

where $\operatorname{Pr}_{i}$ is the conditional probability under hypothesis $H_{i}$. Based on the computed LLR, each sensor makes a local decision $u_{\mathrm{j}}$ regarding the current hypothesis. In the upcoming analysis, we consider two distinct cases for comparison:

\footnotetext{
${ }^{1}$ The analysis presented in the paper can be readily extended to other data models.
}

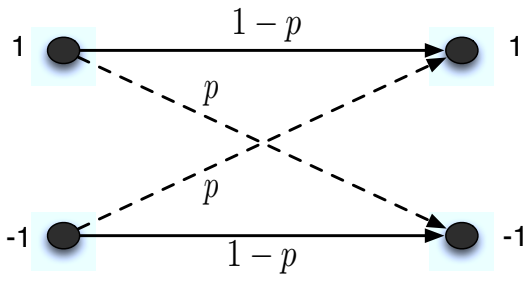

Fig. 2. Binary Symmetric Channel (BSC)

a) Conventional system: A sensor compares $l_{\mathrm{j}}\left(x_{\mathrm{j}}\right)$ to a certain threshold $\tau$, and makes a local decision in favor of $H_{1}$ and transmits the signal $u_{\mathrm{j}}=1$ if $l_{\mathrm{j}}>\tau$, or in favor of $H_{0}$ and transmits $u_{\mathrm{j}}=-1$ if $l_{\mathrm{j}} \leq \tau$. Given the assumed data model, the local decision probabilities given hypothesis $H_{i}, i=0,1$ are given by

$$
\begin{gathered}
\operatorname{Pr}_{i}^{c}\left(u_{\mathrm{j}}=1\right)=2 Q\left(\frac{\sqrt{\eta}}{\sigma_{\mathrm{i}}}\right) \\
\operatorname{Pr}_{i}^{c}\left(u_{\mathrm{j}}=-1\right)=1-2 Q\left(\frac{\sqrt{\eta}}{\sigma_{\mathrm{i}}}\right)
\end{gathered}
$$

where $\operatorname{Pr}_{i}^{c}$ is the conditional probability under hypothesis $H_{i}$ in the conventional case, and

$$
\eta=2\left(\tau-\log \left(\frac{\sigma_{0}}{\sigma_{1}}\right)\right) /\left(\frac{1}{\sigma_{0}^{2}}-\frac{1}{\sigma_{1}^{2}}\right) .
$$

b) Censoring-enabled system: A sensor compares $l_{\mathrm{j}}\left(x_{\mathrm{j}}\right)$ to two different thresholds, namely $\tau_{1}$ and $\tau_{0}$, where $\tau_{1} \geq \tau_{0}$. In this case, the local decisions made by the sensor can be elaborated as follows

$u_{\mathrm{j}}= \begin{cases}1 & \left(l_{\mathrm{j}}>\tau_{1}\right) \\ 0 & \text { No reliable decision can be made }\left(\tau_{0}<l_{\mathrm{j}} \leq \tau_{1}\right) \\ -1 & \left(l_{\mathrm{j}} \leq \tau_{0}\right)\end{cases}$

In this case, given $H_{i}, i=0,1$, the local decision probabilities are given by

$$
\begin{gathered}
\operatorname{Pr}_{i}^{c e n}\left(u_{\mathrm{j}}=1\right)=2 Q\left(\frac{\sqrt{\eta_{1}}}{\sigma_{\mathrm{i}}}\right) \\
\operatorname{Pr}_{i}^{c e n}\left(u_{\mathrm{j}}=-1\right)=1-2 Q\left(\frac{\sqrt{\eta_{0}}}{\sigma_{\mathrm{i}}}\right)
\end{gathered}
$$

where $\operatorname{Pr}_{i}^{c e n}$ is the conditional probability under hypothesis $H_{i}$ in the censoring case, and

$$
\begin{aligned}
& \eta_{1}=\frac{2}{\left(\frac{1}{\sigma_{0}^{2}}-\frac{1}{\sigma_{1}^{2}}\right)}\left(\tau_{1}-\log \left(\frac{\sigma_{0}}{\sigma_{1}}\right)\right) \\
& \eta_{0}=\frac{2}{\left(\frac{1}{\sigma_{0}^{2}}-\frac{1}{\sigma_{1}^{2}}\right)}\left(\tau_{0}-\log \left(\frac{\sigma_{0}}{\sigma_{1}}\right)\right) .
\end{aligned}
$$

\section{B. Global Decision}

The FC sequentially collects the local decisions made by the sensors in order to arrive at a global decision regarding the current hypothesis. System operation can be described as follows. The FC polls a sensor for its local decision, which may be received incorrectly due to wireless channel 


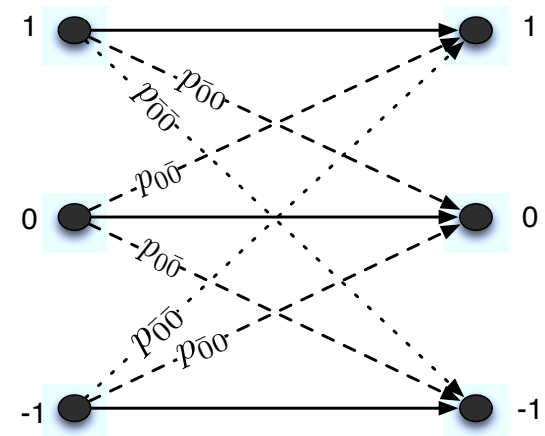

Fig. 3. Ternary Channel

impairment. Based on the received local decisions, the FC decides in favor of either $H_{1}$ or $H_{0}$ if the decision made is reliable enough (enough observations are collected from sensor nodes), or else decides to poll another sensor for its local decision. The process continues until a reliable decision is made by the FC. Accordingly, the decision variable for the FC can be quantitatively expressed as

$$
Z_{\mathrm{J}}=\sum_{\mathrm{j}=1}^{\mathrm{J}} \hat{u}_{\mathrm{j}}
$$

where $\mathrm{J}$ is the total number of polled sensors until a reliable decision is made by the $\mathrm{FC}$, and $\hat{u}_{\mathrm{j}}$ is the received version of the local decision of the jth node that is not necessarily equal to $u_{\mathrm{j}}$. The FC makes a global decision $u_{0}$ regarding the current hypothesis based on the received decisions. We can state the decision process of the $\mathrm{FC}$ as follows:

$$
u_{0}= \begin{cases}\text { Decide in favor of } H_{1} & Z_{\mathrm{J}} \geq \alpha \\ \text { Decide in favor of } H_{0} & Z_{\mathrm{J}} \leq-\beta \\ \text { Request more decisions } & -\beta<Z_{\mathrm{J}}<\alpha\end{cases}
$$

where $\alpha$ and $-\beta$ are predetermined thresholds chosen to satisfy a certain required level of reliability in the global decisions. It is worthy to note here that increasing the magnitude of $\alpha$ and $\beta$ eventually leads to more reliable global decisions at the expense of longer decision times required to poll a sufficient number of sensors to make such decisions.

\section{Wireless Channel}

a) Conventional case: For the conventional case, we model the wireless channel between a sensor and the FC as a binary symmetric channel (BSC) as shown in Fig. 2, in which the transmitted binary signal representing the transmitted local decision may be flipped with a probability $p<0.5$ (i.e., $\left.\operatorname{Pr}\left(\hat{u}_{\mathrm{j}} \neq u_{\mathrm{j}}\right)=p<0.5\right)$. Assuming that the transmitting sensor node employs BPSK modulation with unity-transmitting power, and for an AWGN channel ${ }^{2}$, then we can express the

\footnotetext{
${ }^{2}$ BSC model can be also used for modeling fading channels and the crossover probability will depend on the fading channel model. However, the authors did not see that such a complication in the modeling of the BSC would significantly contribute to the exposition of the main idea of censoring as a performance enhancer.
}

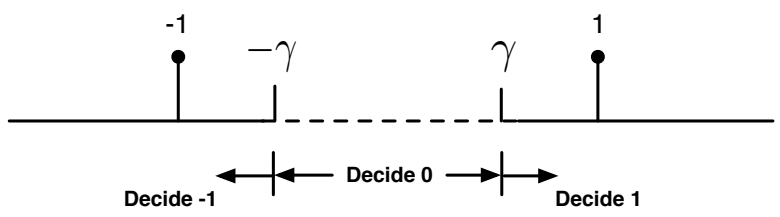

Fig. 4. Detection process at the FC for the ternary channel

transition probability as $p=Q\left(\sigma_{N}^{-1}\right)$, where $\sigma_{N}^{2}$ is the noise variance.

b) Censoring case: For the censoring-enabled systems, we consider two distinct cases: in the first, we assume that by the aid of a "genie", the FC is able to know if the polled sensor decides to refrain from sending a decision (i.e., $u_{\mathrm{j}}=0$ ). If the sensor, however, makes a local decision regarding the current hypothesis, the transmitted signal goes through a BSC similar to the one described previously. We label this scenario as the $F C$-aware system, which - despite being unrealistic - gives an upper bound of the performance of censoring-enabled systems. In the second, which we label the $F C$-unaware system, we assume a more realistic model in which local decisions that are sent to the FC go through a ternary channel as shown in Fig. 3. We assume that the local decision sent by the $j$ th sensor goes through ternay channel where the transition probabilities are $p_{0 \overline{0}}=\operatorname{Pr}(\hat{u}=1 \mid u=0)=\operatorname{Pr}(\hat{u}=-1 \mid u=0)$, $p_{\overline{0} 0}=\operatorname{Pr}(\hat{u}=0 \mid u=-1)=\operatorname{Pr}(\hat{u}=0 \mid u=1)$ and $p_{\overline{00}}=$ $\operatorname{Pr}(\hat{u}=1 \mid u=-1)=\operatorname{Pr}(\hat{u}=-1 \mid u=1)$. We adopt the same transmission scheme and AWGN wireless link between the sensors and the FC. For this case, however, the FC has to detect one of three possible cases, namely transmitting a 1 , transmitting a -1 or censoring transmission. The detection process can be done via comparing to two different thresholds, $\gamma$ and $-\gamma$. The detection process is illustrated in Fig. 4. Accordingly, the transition probabilities can be written as follows

$$
\begin{aligned}
& p_{0 \overline{0}}=Q\left(\frac{\gamma}{\sigma_{N}}\right), p_{\overline{0} 0}=Q\left(\frac{1-\gamma}{\sigma_{N}}\right)-Q\left(\frac{1+\gamma}{\sigma_{N}}\right) \\
& p_{\overline{00}}=Q\left(\frac{1+\gamma}{\sigma_{N}}\right)
\end{aligned}
$$

In the next sections, we analyze the described systems in terms of average error probabilities and the average incurred delay (i.e., the average number of observations needed until a decision is made at the FC), and we characterize the effect of enabling censoring on the overall system performance in terms of these metrics.

\section{RANDOM WALKS}

In this section, we provide the mathematical basis for the upcoming performance analysis of the aforementioned detection systems.

\section{A. Average Error Probability}

In this section, we characterize the average error probability of the global decision. By examining (6), it is clear that the stochastic process $\left\{Z_{n}, n>1\right\}$ is a one-dimensional simple 
random walk with two absorbing barriers based on $\hat{u}_{\mathrm{j}}, \mathrm{j}=$ $1, \ldots, n[14]$. Each of the variables $\hat{u}_{\mathrm{j}}, \mathrm{j}=1, \ldots, n$ changes the summation $Z_{n}$ by either +1 or -1 , and the process continues until one of two thresholds is crossed, either $Z_{n} \geq \alpha$ or $Z_{n} \leq$ $-\beta$, in which case a global decision is made in favor of $H_{1}$ or $H_{0}$ respectively. An error occurs if given $H_{1}, Z_{n}$ crosses $\alpha$, or given $H_{0}, Z_{n}$ crosses $-\beta$. For arbitrary $n \geq \alpha$, Chernoff bound can be used to find an exponentially-tight bound on the probability of the summation $Z_{n}$ crossing a threshold $\alpha$ [14]. Let $p_{1}^{(\mathrm{i})}=\operatorname{Pr}_{\mathrm{i}}\left(\hat{u}_{\mathrm{j}}=1\right)$ and $p_{-1}^{(\mathrm{i})}=\operatorname{Pr}_{\mathrm{i}}\left(\hat{u}_{\mathrm{j}}=-1\right)$. Given hypothesis $H_{0}$, and assuming that $E_{0}\left(\hat{u}_{\mathrm{j}}\right)<0^{3}$, this bound is given by

$$
\operatorname{Pr}_{0}\left(Z_{n} \geq \alpha\right) \leq \exp \left[\alpha\left(\frac{\gamma\left(r_{0}\right)}{\grave{\gamma}\left(r_{0}\right)}-r_{0}\right)\right] \leq \exp \left(-r^{\star} \alpha\right)
$$

where $\grave{\gamma}\left(r_{0}\right)=\alpha / n, \gamma(r)=\ln g_{\hat{\mathrm{U}}}(r), g_{\hat{\mathrm{U}}}(r)=E\left(e^{(r \hat{\mathrm{U}})}\right)$ is the Moment Generating Function of the random variable $\hat{\mathrm{U}}$, and $r^{\star}$ is the solution to the equation $\gamma(r)=0$. Chernoff bound then gives an upper bound on the error probability conditioned on $H_{0}$ by considering the probabilities $p_{1}^{(\mathrm{i})}$ and $p_{-1}^{(\mathrm{i})}$ for each of the prescribed systems. To find an upper bound on the errror probability conditioned on $H_{1}$, we observe the simple random walk $\left\{-Z_{\mathrm{n}}, \mathrm{n}>1\right\}$, assuming that $E_{1}\left(\hat{u}_{\mathrm{j}}\right)>0$. In this case, applying the Chernoff bound yields

$$
\operatorname{Pr}_{1}\left(Z_{\mathrm{n}} \leq \beta\right) \leq \exp \left[-\beta\left(\frac{\gamma\left(r_{0}\right)}{\grave{\gamma}\left(r_{0}\right)}-r_{0}\right)\right] \leq \exp \left(-r^{\star} \beta\right)
$$

\section{B. Average Stopping Time}

In this section, we characterize the average number of local decisions required for a reliable global decision. Considering the simple random walk represented by $\left\{Z_{n}, n>1\right\}$, we aim at obtaining upper bounds on the conditional average stopping time of its stopping trial $\mathrm{J}$.

Let $\mathrm{J}$ be the value of $n$ in (9) at which a threshold is first crossed. Then by virtue of the following lemma, $\mathrm{J}$ is a stopping trial for $Z_{n}$.

Lemma III.1. Let $\left\{X_{i} ; i \geq 1\right\}$ be IID rvs, not identically 0 . For each $n \geq 1$, let $S_{n}=X_{1}+\ldots+X_{n}$. Let $\alpha>0$ and $\beta>0$ be arbitrary, and let $J$ be the smallest $n$ for which either $S_{n} \geq \alpha$ or $S_{n} \leq-\beta$. Then $J$ is a random variable (i.e., $\lim _{m \rightarrow \infty} \operatorname{Pr}(J \geq m)=0$ ) and has finite moments of all orders.

Proof: Refer to [14].

Wald's equality [15] relates the average stopping time $E(\mathrm{~J})$ of the random walk in (6) to the expected values of the random variables $\hat{u}_{\mathrm{j}}$ and their summation as follows

$$
E_{i}\left(Z_{\mathrm{J}}\right)=E_{i}(\mathrm{~J}) E_{i}(\hat{u})
$$

where $E_{i}$ denotes conditional expectation under $H_{i}$, and the subscript of $\hat{u}_{\mathrm{j}}$ in $E_{i}(\hat{u})$ has been dropped due to the fact

\footnotetext{
${ }^{3}$ To ensure the validity of the condition, the values of the used local thresholds $\left(\eta\right.$ or $\left.\eta_{0}, \eta_{1}\right)$ must satisfy $\left.p_{1}^{(\mathrm{i})}<p_{-1}^{(\mathrm{i})}\right\}$
}

that given $H_{i}, i=0,1, \hat{u}_{\mathrm{j}}, \mathrm{j}=1, \ldots, \mathrm{J}$ are IID. Given $H_{0}$, a stopping trial ends with a correct decision if $Z_{\mathrm{J}}=-\beta$, or with an erroneous decision if $Z_{\mathrm{J}}=\alpha$ (here, we neglect the overshoots in $Z_{\mathrm{J}}$ assuming integer values for $\alpha$ and $\beta$ ). Therefore, we can write the conditional expected value of $Z_{\mathrm{J}}$ as

$$
\begin{aligned}
E_{0}\left(Z_{\mathrm{J}}\right) & =\alpha \operatorname{Pr}_{0}\left(Z_{n} \geq \alpha\right)+(-\beta)\left[1-\operatorname{Pr}_{0}\left(Z_{n} \geq \alpha\right)\right] \\
& =-\beta+(\alpha+\beta) \operatorname{Pr}_{0}\left(Z_{n} \geq \alpha\right)
\end{aligned}
$$

and conversely we can write the expected value of $Z_{\mathrm{J}}$ conditioned on $H_{1}$ as

$$
\begin{aligned}
E_{1}\left(Z_{\mathrm{J}}\right) & =(-\beta) \operatorname{Pr}_{1}\left(Z_{n} \leq-\beta\right)+\alpha\left[1-\operatorname{Pr}_{1}\left(Z_{n} \leq-\beta\right)\right] \\
& =\alpha-(\alpha+\beta) \operatorname{Pr}_{1}\left(Z_{n} \leq-\beta\right) .
\end{aligned}
$$

Considering the probabilities $p_{1}^{(\mathrm{i})}$ and $p_{-1}^{(\mathrm{i})}$ for each of the detection systems, we can find an upper bound on the conditional expected number of observations $E_{i}(\mathrm{~J})$.

\section{Performance Analysis}

In this section, we develop closed form expressions that characterize the performance of the prescribed systems in terms of the error probability of the global decision and the expected incurred delay (expected number of observations until a global decision is made).

\section{A. Conventional System}

We consider the conventional system model of a sequential detection network, in which a sensor sends its local decision when polled by the FC. In this case, the values of $p_{1}^{(\mathrm{i})}$ and $p_{-1}^{(\mathrm{i})}$ become

$$
\begin{aligned}
& p_{1}^{(\mathrm{i})}=2 Q\left(\frac{\sqrt{\eta}}{\sigma_{\mathrm{i}}}\right)(1-p)+\left(1-2 Q\left(\frac{\sqrt{\eta}}{\sigma_{\mathrm{i}}}\right)\right) p, \\
& p_{-1}^{(\mathrm{i})}=1-p_{1}^{(\mathrm{i})} .
\end{aligned}
$$

Applying Chernoff bound (see Appendix A) then yields an upper bound on the error probability conditioned on $H_{0}$ and $H_{1}$ as

$$
P_{\mathcal{E} / H_{0}} \leq\left(\frac{1-p_{\mathrm{u}}^{(0)}}{p_{\mathrm{u}}^{(0)}}\right)^{-\alpha}, P_{\mathcal{E} / H_{1}} \leq\left(\frac{p_{\mathrm{u}}^{(1)}}{1-p_{\mathrm{u}}^{(1)}}\right)^{-\beta}
$$

The average number of observations needed for a global decision conditioned on respectively $H_{0}$ and $H_{1}$ can be given by

$$
\begin{aligned}
& E_{0}(\mathrm{~J}) \leq \frac{-\beta+(\alpha+\beta)\left(\frac{1-p_{\mathrm{u}}^{(0)}}{p_{\mathrm{u}}^{(\mathrm{o})}}\right)^{-\alpha}}{2 p_{u}^{(0)}-1}, \\
& E_{1}(\mathrm{~J}) \leq \frac{\alpha-(\alpha+\beta)\left(\frac{p_{\mathrm{u}}^{(1)}}{1-p_{\mathrm{u}}^{(1)}}\right)^{-\beta}}{2 p_{u}^{(1)}-1}
\end{aligned}
$$




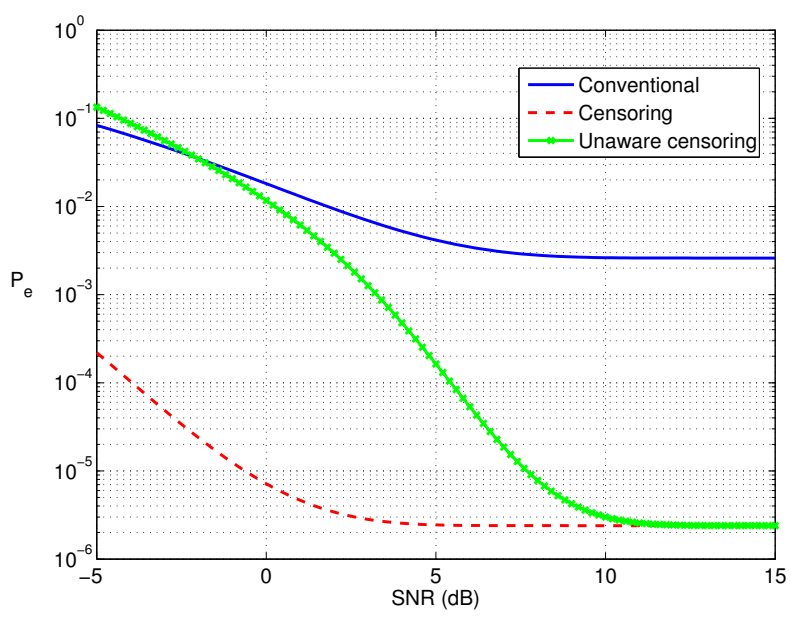

Fig. 5. Bound on the error probability for all systems

\section{B. FC-aware}

We first study the FC-aware case, in which the FC knows beforehand if the polled sensor censors transmission, and we characterize the error probability and the average stopping time in this case. Note that the FC-aware system gives a benchmark performance of censoring-enabled systems in general in terms of average stopping time. We assume that the FC is able to determine if a sensor decides locally to refrain from sending. Based on that assumption, we can adopt the same BSC between the nodes and the FC. By finding the values of $p_{1}^{(\mathrm{i})}$ and $p_{-1}^{(\mathrm{i})}$, we can derive the upper bounds for the conditional average error probabilities and the average number of observations, which are found to be equal to of the same functional form of (16) and (17) respectively, but with values of $p_{1}^{(\mathrm{i})}$ and $p_{-1}^{(\mathrm{i})}$ obtained for this case.

\section{FC-unaware}

We now consider the more realistic model where the local decisions are transmitted through a ternary channel as discussed in Section II. Similar analysis to the previous subsection can be applied based on the values of $p_{1}^{(\mathrm{i})}$ and $p_{-1}^{(\mathrm{i})}$ in this case. The upper bounds of the conditional error probabilities and the average number of observations are found to be equal to (18) and (20) respectively (see Appendix B)

$$
\begin{gathered}
P_{\mathcal{E} / H_{0}} \leq\left(\frac{p_{-1}^{(0)}}{p_{1}^{(0)}}\right)^{-\alpha}, P_{\mathcal{E} / H_{1}} \leq\left(\frac{p_{1}^{(1)}}{p_{-1}^{(1)}}\right)^{-\beta} \\
E_{0}\left(Z_{\mathrm{J}}\right) \leq-\beta+(\alpha+\beta)\left(\frac{\bar{p}_{-1}^{(0)}}{\bar{p}_{1}^{(0)}}\right)^{-\alpha} \\
E_{1}\left(Z_{\mathrm{J}}\right) \leq \alpha-(\alpha+\beta)\left(\frac{\bar{p}_{1}^{(1)}}{\bar{p}_{-1}^{(1)}}\right)^{-\beta} \\
E_{0}(\mathrm{~J}) \leq \frac{E_{0}\left(Z_{\mathrm{J}}\right)}{p_{1}^{(0)}-p_{-1}^{(0)}}, E_{1}(\mathrm{~J}) \leq \frac{E_{1}\left(Z_{\mathrm{J}}\right)}{p_{1}^{(1)}-p_{-1}^{(1)}}
\end{gathered}
$$

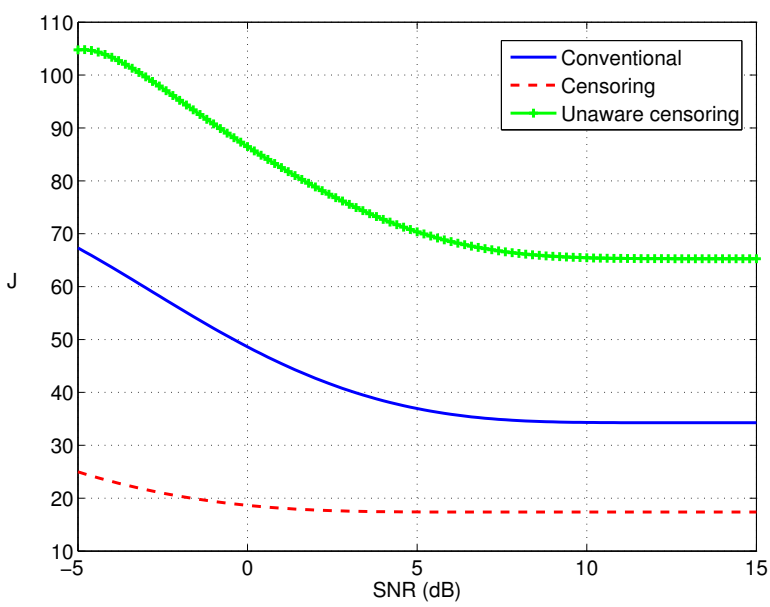

Fig. 6. Bound on the expected number of observations for both systems

\section{Numerical Evaluation}

In this section, we numerically evaluate the performance of both the conventional and the censoring-enabled systems ${ }^{4}$. We assume that a certain level of confidence in the global decision is to be maintained, which effectively translates to $\alpha=\beta=10$ for all three systems. We assume that $\sigma_{1}=1$ and $\sigma_{0}=0.25$, and $\gamma=0.5$. System performance is measured against the SNR at the FC sensing channel, defined as SNR = $\log \left(P / \sigma_{N}^{2}\right)$ where $P$ is the average received power by the FC from a local sensor and is set to $P=1$.

Figures 5 and 6 show the error probability and the average stopping time for the conventional and both the censoring cases. Fig. 6 clearly indicates that the conventional system gives superior performance in terms of average delay than FC-unaware censoring systems, while FC-aware system outperforms them both. On the other hand, for the average error probability, Fig. 5 gives a different view of the three systems. It is clear that FC-aware system also outperforms both the conventional and FC-unaware systems for all values of SNR. However, neither the conventional nor the FC-unaware system dominates the other for all SNR regions. In specific, for the low SNR regime, the conventional system outperforms the FC-unaware system, while the latter provides higher rate of improvement than the former when increasing SNR. In fact, the FC-unaware system achieves the optimal performance of the FC-aware system in the high SNR regime, while maintaining a better performance level than the conventional system in the mid SNR regime.

Combining the observations made from both Fig. 5 and Fig. 6 , we come to the following conclusions regarding conventional and censoring systems:

- FC-aware system performance is superior to conventional system in terms of both error probability and average delay.

\footnotetext{
${ }^{4}$ Numerical simulations were not provided since we derive upper bounds for the probability of rare events, which renders numerical simulations infeasible.
} 


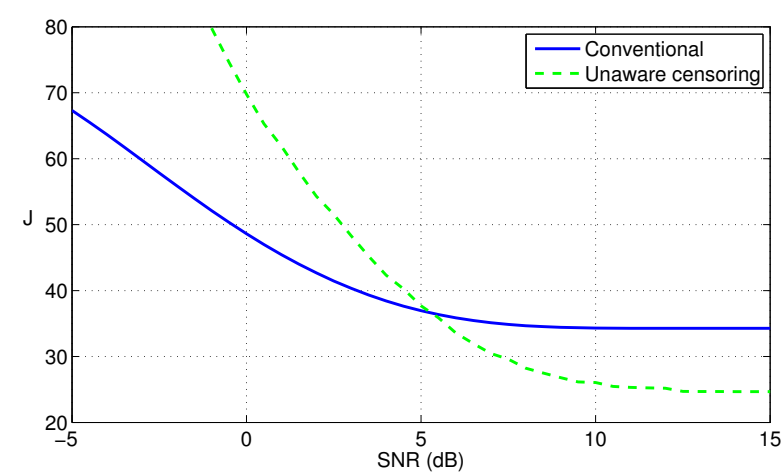

Fig. 7. Average stopping time for the conventional system and the FCunaware system with fixed error probability

- FC-unaware system performance is inferior to the conventional system in terms of average delay.

- In terms of error probability, conventional system outperforms the FC-unaware system in the low SNR regime, and vise versa in the mid and high SNR regimes.

While it is unquestionable that in terms of error probability and average delay, censoring should not be employed in the low SNR regime, it is unclear whether one should employ censoring in the mid and high SNR regimes given the contradicting influences on both performance metrics.

To provide a justifiable answer to the latter question, we consider a set of two modified scenarios for comparison.

1) In the first, we deliberately modify $\alpha$ and $\beta$ for the FC-unaware system to give a matching performance - in terms of error probability - to the conventional case with fixed $\alpha=\beta=10$ as in the previous case. For this scenario, we then compare the average delay incurred in both systems as shown in Fig. 7. It is clear that for a fixed error probability, FC-aware system provides better performance in the high SNR region (nearly greater than $\mathrm{SNR}=5$ ), which indicates the region where censoring should be employed for better system performance. Needless to say, the SNR value which marks the beginning of the high SNR regime is a function of the values of $\alpha$ and $\beta$ pertained to a required level of performance.

2) In the second, we modify $\alpha$ and $\beta$ for the conventional system to give a matching performance - in terms of average delay - to the FC-unaware case with fixed $\alpha=$ $\beta=10$. For this scenario, we then compare the error probability in both systems as shown in Fig. 8. It is shown that censoring provides better performance in the high SNR regime, which conforms with the observation from the previous scenario.

Finally, it is worth mentioning that a typical WSN does not exhibit the same channel characteristics for a prolonged time period. Conversely, the received SNR perceived by the FC may vary over the channel coherence time. It is therefore expected for a WSN to alternate randomly between high, mid and low SNR regimes.

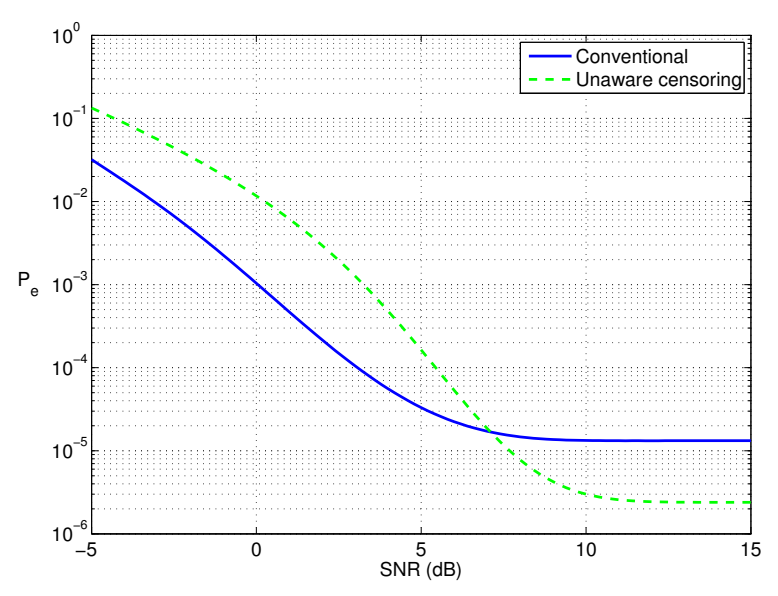

Fig. 8. Error probability for the conventional system and the FC-unaware system with equal average stopping time

In this case, a FC should undergo a periodic channel estimation phase to determine the region in which the system is likely to operate, and accordingly decide the mode of operation: to employ censoring or not. The FC then informs sensor nodes with the mode of operation via low-cost control packets, or piggybacked on existing control packets that are used for network calibration and synchronization.

\section{CONCLUSION}

We considered sequential distributed detection in WSNs where the FC collects the local decisions from the sensor nodes in a sequential manner. A comparison was held between the conventional system and two censoring-enabled systems, namely the FC-aware and the FC-unaware systems. Based on the theory of Random Walks, an analytical characterization of the performance for the three systems was made in terms of the error probability of the global decision made by the FC, and the average number of observations needed until such a decision is reached. Numerical examples were also provided to highlight the key observation that in the low SNR regimes, it is better in terms of the error probability and the average stopping time to employ conventional detection, while it is optimal in the high SNR regimes to employ the FC-unaware censoring scheme.

\section{APPENDIX}

\section{A. Upper bound on error proability in the conventional case}

We aim at obtaining a closed-form expression for $r^{\star}$, which is the solution for the equation $\gamma(r)=0$ in (9).

Proof: Since $\gamma(r)=\ln g_{\hat{U}}(r)$, then $\gamma(r)=0$ translates to $g_{\hat{U}}(r)=1 . g_{\hat{U}}(r)$ can be written as

$$
g_{\hat{U}}(r)=p_{\mathrm{u}}^{(0)} e^{r}+\left(1-p_{\mathrm{u}}^{(0)}\right) e^{-r} .
$$

It is clear that $g_{\hat{U}}(r)=1$ is a quadratic equation in $e^{r}$ which when solved yields the two solutions $e^{r^{\star}}=1$ and $e^{r^{\star}}=\left(\frac{1-p_{\mathrm{u}}^{(0)}}{p_{\mathrm{u}}^{(0)}}\right)$, the former of which is trivial and thus 
neglected. Taking the natural logarithm of $e^{r^{\star}}$ therefore gives $r^{\star}=\ln \left(\frac{1-p_{\mathrm{u}}^{(0)}}{p_{\mathrm{u}}^{(0)}}\right)$.

\section{B. Upper bound on error probability in the unaware censoring} case

We aim at finding an upper bound on the error probability for the FC-unaware censoring case given $H_{0}$ and $H_{1}$, as expressed in (18).

Proof: According to Chernoff bound as stated in (9), the upper bound on the error probability given $H_{0}$ is a function of $r^{\star}$ which is the solution to $\gamma(r)=0$. Similar to Appendix A, we find $r^{\star}$ by equivalently solving the quadratic equation $g_{\hat{U}}(r)=1$ in $e^{r}$. In the FC-unaware case, $g_{\hat{U}}(r)$ is expressed as

$$
g_{\hat{U}}(r)=\bar{p}_{1}^{(0)} e^{r}+\bar{p}_{-1}^{(0)} e^{-r}+\left(1-\bar{p}_{1}^{(0)}-\bar{p}_{-1}^{(0)}\right)
$$

Solving $g_{\hat{U}}(r)=1$ yields the solution $r^{\star}=\ln \left(\frac{\bar{p}_{-1}^{(0)}}{\bar{p}_{1}^{(0)}}\right)$. By plugging this value into (9), we obtain the first bound in (18). Following a similar straightforward analysis, we can obtain the second bound in (18).

\section{REFERENCES}

[1] R.S. Blum, S.A. Kassam, and H.V. Poor, "Distributed detection with multiple sensors i. advanced topics," Proceedings of the IEEE, vol. 85, no. 1, pp. 64-79, 1997.

[2] C. Rago, P. Willett, and Y. Bar-Shalom, "Censoring sensors: A lowcommunication-rate scheme for distributed detection," IEEE Transactions on Aerospace and Electronic Systems, vol. 32, no. 2, pp. 554-568, April 1996.

[3] Swaroop Appadwedula, Venugopal V Veeravalli, and Douglas L Jones, "Energy-efficient detection in sensor networks," IEEE Journal on Selected Areas in Communications, vol. 23, no. 4, pp. 693-702, 2005.

[4] W.P. Tay, J.N. Tsitsiklis, and M.Z. Win, "Censoring sensors: Asymptotics and the value of cooperation," in 40th Annual Conference on Information Sciences and Systems, Princeton, NJ, March 2006.

[5] S. Appadwedula, V.V. Veeravalli, and D.L. Jones, "Decentralized detection with censoring sensors," IEEE Transactions on Signal Processing, vol. 56, no. 4, pp. 1362-1373, April 2008.

[6] J.F. Chamberland and V.V. Veeravalli, "Decentralized detection in sensor networks," IEEE Transactions on Signal Processing, vol. 51, no. 2, pp. 407-416, 2003.

[7] Sachin Chaudhari et al., "Spectrum sensing for cognitive radios: Algorithms, performance, and limitations," 2012.

[8] Maryam Najimi, Ataollah Ebrahimzadeh, S. Mehdi Hosseini Andargoli, and Afshin Fallahi, "A novel sensing nodes and decision node selection method for energy efficiency of cooperative spectrum sensing in cognitive sensor networks," IEEE Sensors Journal, vol. 13, no. 5, 2013.

[9] Jithin Kazuthuveettil Sreedharan and Vinod Sharma, "A novel algorithm for cooperative distributed sequential spectrum sensing in cognitive radio," in IEEE Wireless Communications and Networking Conference, WCNC 2011, pp. 1881-1886.

[10] K. G. Seddik, A. K. Sultan, and A. El-Sherif, "Censoring for improved performance of distributed detection in wireless sensor networks," in IEEE Workshop on Signal Processing Advances in Wireless Communications (SPAWC), San Francisco, CA, June 2011.

[11] M. Karmoose, K.G. Seddik, and H. El Kamchouchi, "Censoring for type-based multiple access scheme in wireless sensor networks," in IEEE Vehicular Technology Conference, VTC 2012, pp. 1-6.

[12] V.V. Veeravalli, T. Basar, and H.V. Poor, "Decentralized sequential detection with a fusion center performing the sequential test," IEEE Transactions on Information Theory, vol. 39, no. 2, pp. 433-442, 1993.

[13] HR Hashemi and I.B. Rhodes, "Decentralized sequential detection," IEEE Transactions on Information Theory, vol. 35, no. 3, pp. 509-520, 1989.
[14] R.G. Gallager, Discrete stochastic processes, vol. 101, Kluwer Academic Publishers, 1996.

[15] Abraham Wald, "On cumulative sums of random variables," The Annals of Mathematical Statistics, pp. 283-296, 1944. 